\title{
A GENERALIZATION TO MULTIFUNCTIONS OF FAN'S BEST APPROXIMATION THEOREM
}

\author{
V. M. SEHGAL AND S. P. SINGH \\ (Communicated by Paul S. Muhly)
}

\begin{abstract}
We prove a theorem for set valued mappings in an approximatively compact, convex subset of a locally convex space, and then derive results due to Ky Fan and S. Reich as corollaries.
\end{abstract}

Let $E$ be a locally convex Hausdorff topological vector sapce, $S$ a nonempty subset of $E$ and $p$ a continuous seminorm on $E$. It is a well-known result (see the proof in Sehgal [8] or Ky Fan [1]) that if $S$ is compact and convex and $f: S \rightarrow E$ is a continuous map, then there exists an $x \in S$ satisfying

$$
p(f x-x)=d_{p}(f x, S)=\min \{p(f x,-y) \mid y \in S\} .
$$

Since then a number of authors have provided either an extension of the above theorem to set valued mappings or have weakened the compactness condition therein. Some of these results are

(a) REICH (1978). If $S$ is approximatively compact and $f: S \rightarrow E$ is continuous with $f(S)$ relatively compact, then (1) holds [5].

(b) LIN (1979). If $S$ is a closed unit ball of a Banach space $X$ and $f: S \rightarrow X$ is a continuous condensing map, then (1) holds when $p$ is the norm on $X$ [4].

(c) WATERS (1984). If $S$ is a closed and convex subset of a uniformly convex Banach space $E$ and $f: S \rightarrow 2^{E}$ is a continuous multifunction with convex and compact values and $f(S)$ is relatively compact, then (1) holds [9].

(d) SEHGAL AND SINGH (1985). Let $S \subseteq E$ with $\operatorname{int}(S) \neq \varnothing$ and $\operatorname{cl}(S)$ convex and let $f: S \rightarrow 2^{E}$ be a continuous condensing multifunction with convex, compact values and with a bounded range. Then for each $w \in \operatorname{int}(S)$, there exists a continuous seminorm $p=p(w)$ satisfying (1) [6].

Our aim in this presentation is to prove (a) for multifunctions and derive some results as easy corollaries.

For definitions and terminologies we refer to Reich [5] (see also [3]).

DEFINITION. A subset $S$ of $E$ is approximatively $p$-compact iff for each $y \in E$ and a net $\left\{x_{\alpha}\right\}$ in $S$ satisfying $p\left(x_{\alpha}-y\right) \rightarrow d_{p}(y, S)$ there is a subnet $\left\{x_{\beta}\right\}$ and an $x \in S$ such that $x_{\beta} \rightarrow x$.

Clearly a compact set in $E$ is approximatively compact. The converse, however, may fail. For example, the closed unit ball of an infinite dimensional uniformly convex Banach space is approximatively norm compact but not compact.

Received by the editors September 16, 1985 and, in revised form, November 17, 1986. 1980 Mathematics Subject Classification (1985 Revision). Primary 47H10; Secondary 54H25.

Key words and phrases. Approximately compact set, multivalued mappings, upper and lower semicontinuous mappings, condensing map, uniformly convex.

This work, in part, was supported by NSERC and NATO Grants. 
Some consequences of the definition follow.

1. An approximatively $p$-compact set $S$ in $E$ is closed. Let $y$ be a cluster point of $S$ and let a net $\left\{x_{\alpha}\right\} \subseteq S$ satisfy $p\left(x_{\alpha}-y\right) \rightarrow d_{p}(y, S)=0$. Since $S$ is approximatively $p$-compact, $\left\{x_{\alpha}\right\}$ contains a subnet $x_{\beta} \rightarrow x \in s$. Since $x_{\beta} \rightarrow y$ also and $E$ is Hausdorff, $x=y \in S$.

2. If $S$ is a closed and convex subset of a uniformly convex Banach space then $S$ is approximaltively norm compact.

Let $y \in E$ and, without loss of generality, assume a sequence $\left\{x_{n}\right\} \subseteq S$ satisfies $\left\|x_{n}-y\right\| \rightarrow d(y, S) \equiv \inf \{\|y-x\| \mid x \in S\}$. This implies that $\sup \left\|x_{n}\right\|<\infty$. Consequently, since $S$ is closed and convex, there exist an $x \in S$ and a subsequence $\left\{x_{n_{i}}\right\}$ of the sequence $\left\{x_{n}\right\}$ such that $x_{n_{i}} \rightarrow x$ weakly. Thus

$$
x_{n_{i}}-y \rightarrow x-y \text { weakly. }
$$

It follows from $(*)$ that

$$
\|x-y\| \leq \lim \left\|x_{n_{i}}-y\right\|=d(y, S),
$$

i.e. $d(y, S)=\|x-y\|$.

Consequently, by the definition of the sequence $\left\{x_{n}\right\}$

$$
\left\|x_{n_{i}}-y\right\| \rightarrow\|x-y\| .
$$

Since $E$ is uniformly convex, (*) and (**) imply that $x_{n_{i}}-y \rightarrow x-y$. This yields $x_{n_{i}} \rightarrow x \in S$. Thus $S$ is approximatively norm compact.

DEFINITION. Let $E$ and $F$ be topological vector spaces and let $2^{F}$ denote the family of nonempty subsets of $F$. The mapping $T: E \rightarrow 2^{F}$ is upper semicontinuous (u.s.c.) iff $T^{-1}(B)=\{x \in E \mid T x \cap B \neq \varnothing\}$ is closed for each closed subset $B$ of $F$.

3. If $S$ is an approximatively $p$-compact subset of $E$ then for each $y \in E$, $Q(y)=\left\{x \in S \mid p(y-x)=d_{p}(y, S)\right\}$ is nonempty and the mapping defined by $y \rightarrow Q(y)$ is an upper semicontinuous (u.s.c.) multifunction on $E$. For a proof see Reich [5].

Note that if $E$ is a uniformly convex Banach space the above projection map $Q$ is single valued and continuous.

Now we give our main result.

THEOREM 1. Let $S$ be an approximatively p-compact, convex subset of $E$ and let $F: S \rightarrow 2^{E}$ be a continuous multifunction with closed and convex values. If $F S=\bigcup\{F x \mid x \in S\}$ is relatively compact then there exists an $x \in S$ with

$$
d_{p}(x, F x)=d_{p}(F x, S) .
$$

Further, if $d_{p}(x, F x)>0$, then $x \in \partial S$.

Note that $d_{p}(A, B)=\inf \{p(x-y) \mid x \in A, y \in B\}$.

The proof of the above theorem uses the following lemma, whose proof is given in Sehgal and Singh [7, Lemma 2, p. 92].

LEMMA. Under the hypotheses of Theorem 1, the mapping $g: S \rightarrow R$ (reals) defined by $g(x)=d_{p}(F x, S)$ is continuous.

ProOF OF THEOREM 1. Define a mapping $G: S \rightarrow 2^{S}$ by

$$
G(x)=\bigcup\left\{Q(y) \mid y \in F x, d_{p}(F x, S)=d_{p}(y, S)\right\} .
$$

Note that since $F x$ is compact, $G(x) \neq \varnothing$. 
Further, since $F x$ is convex, it follows that $G x$ is also convex. In fact, if $u$ and $v$ are in $G x$, then there exist elements $y_{1}$ and $y_{2}$ in $F x$ such that $u$ is in $F y_{1}$ and $v$ is in $F y_{2}$ and

$$
p\left(y_{1}-u\right)=d_{p}(y, S)=d_{p}(F x, S)=d_{p}\left(y_{2}, S\right)=p\left(y_{2}-v\right) .
$$

Let $t \in[0,1], w(t)=t u+(1-t) v$ and $y_{3}=t y_{1}+(1-t) y_{2}$. Then $w(t) \in S, y_{3}$ is in $F x$ and

$$
\begin{aligned}
d_{p}\left(y_{3}, S\right) & \leq p\left(y_{3}-w(t)\right) \leq t p\left(y_{1}-u\right)+(1-t) p\left(y_{2}-v\right) \\
& =d_{p}(F x, S) \leq d_{p}\left(y_{3}, S\right) .
\end{aligned}
$$

This implies that

$$
d_{p}\left(y_{3}, S\right)=p\left(y_{3}-w(t)\right)=d_{p}(F x, S) .
$$

Consequently it follows that for any $t \in[0,1]$,

$$
w(t) \in Q\left(y_{3}\right) \cap G x
$$

that is, $G x$ is convex.

Also, since for each $x \in S$,

$$
G x=Q F x \cap\left\{y \in F x \mid d_{p}(F x, S)=d_{p}(y, S)\right\},
$$

and $Q$ is an u.s.c. function, it follows that $G x$ is a closed (in fact, compact) subset of $S$.

We show that $G$ is an u.s.c. multifunction. To prove this, we show that $G^{-1}(A)$ is closed for any closed subset $A$ of $S$. Let $\left\{x_{\alpha}\right\} \subseteq G^{-1}(A)$ be a net such that $x_{\alpha} \rightarrow x_{0} \in S$. Since $G\left(x_{\alpha}\right) \cap A \neq \varnothing$, choose for each $\alpha, z_{\alpha} \in G x_{\alpha} \cap A$. It then follows from the definition of $G$ that for each $\alpha$, there is a $y_{\alpha} \in F x_{\alpha}$, with $d_{p}\left(F x_{\alpha}, S\right)=$ $d_{p}\left(y_{\alpha}, S\right)$ and $z_{\alpha} \in Q\left(y_{\alpha}\right)$. Since $\operatorname{cl}(F S)$ is compact and $\left\{y_{\alpha}\right\} \subseteq F S$, without loss of generality we may assume that $y_{\alpha} \rightarrow y_{0} \in E$. Further, $F$ being u.s.c., it follows that $y_{0} \in F x_{0}$. Also, since $Q$ is u.s.c., $Q(\operatorname{cl}(F S))$ is compact and since for each $\alpha$, $z_{\alpha} \in Q\left(y_{\alpha}\right) \subseteq Q\left(F x_{\alpha}\right) \subseteq Q(\operatorname{cl}(F S))$, we may again assume $z_{\alpha} \rightarrow z_{0} \in Q\left(y_{0}\right)$. Now, $d_{p}\left(y_{\alpha}, S\right) \rightarrow d_{p}\left(y_{0}, S\right)$ and by the lemma $d_{p}\left(F x_{\alpha}, S\right) \rightarrow d_{p}\left(F x_{0}, S\right)$. This implies that $d_{p}\left(y_{0}, S\right)=d_{p}\left(F x_{0}, S\right)$ and that $z_{0} \in G\left(x_{0}\right) \cap A$, i.e., $x_{0} \in G^{-1}(A)$. Thus $G$ is u.s.c. It now follows by a theorem of Himmelberg [2] that there is an $x \in S$ with $x \in G(x)$. This implies that $x \in Q(y)$ for some $y \in F x$ with $d_{p}(F x, S)=d_{p}(y, S)$. Now, since $d_{p}(x, F x) \leq p(x-y)=d_{p}(y, S)=d_{p}(F x, S) \leq d_{p}(x, F x)$, we have $d_{p}(x, F x)=d_{p}(F x, S)$.

If $d_{p}(x, F x)>0$ then $F x \cap S=\varnothing$. Choose a point $y \in F x$ such that $d_{p}(x, F x)=$ $p(x-y)$. If $x$ is an interior point of $S$, then the convexity of $S$ implies the existence of a $z \in \partial S$ such that $p(z-y)<d_{p}(x, F x)$. This implies that $d_{p}(F x, S) \leq p(z-y)<$ $d_{p}(x, F x)$, which gives a contradiction. Consequently in this case $x \in \partial S$.

Note that in view of consequence (2), the result due to Waters is a special case of Theorem 1.

The following simple example is due to Waters [9] and shows that even in the special case of the uniformly convex Banach space $E$, continuity therein cannot be replaced by u.s.c. alone.

EXAMPLE. Let $E=R^{2}$ with the Euclidean norm and let $S=[0,1] \times\{0\}$. Clearly $S$ is convex and compact. 
Define $F: S \rightarrow 2^{E}$ by

$$
F(a, 0)=\left\{\begin{array}{l}
(0,1) \quad \text { if } a \neq 0, \\
L=\text { the line segment }[(0,1),(1,0)] \text { if } a=0 .
\end{array}\right.
$$

Then for any $A \subseteq E$,

$$
F^{-1}(A)=\left\{\begin{array}{l}
\phi \quad \text { if } A \cap L=\varnothing, \\
S \quad \text { if }(0,1) \in A \\
(0,0) \text { if }(0,1) \notin A, A \cap L \neq \varnothing .
\end{array}\right.
$$

Thus $F$ is an u.s.c. but not a l.s.c. multifunction and $F S$ is compact.

However, for any $(a, 0)$,

$$
\begin{aligned}
d((a, 0), F(a, 0))>1 & =d(F(a, 0), S) \quad \text { if } a \neq 0, \\
& =\frac{\sqrt{2}}{2} \neq d(F(0,0), S)=0 \quad \text { if } a=0 .
\end{aligned}
$$

Thus $F$ does not satisfy the conclusion of Theorem 1 .

ACKNOWLEDGMENT. The authors thank the referee for his suggestions improving both the presentation and the contents of this paper.

\section{REFERENCES}

1. Ky Fan, Extensions of two fixed point theorems of F. E. Browder, Math. Z. 112 (1969), 234-240.

2. C. J. Himmelberg, Fixed points of compact multifunctions, J. Math. Anal. Appl. 38 (1972), 205-207.

3. J. L. Kelley and I. Namioka, Linear topological spaces, Van Nostrand, Princeton, N.J., 1953.

4. T. C. Lin, A nopte on a theorem of Ky Fan, Canad. Math. Bull. 22 (1979), 513-515.

5. S. Reich, Approximate selections, best approximations, fixed points and invariant sets, J. Math. Anal. Appl. 62 (1978), 104-113.

6. V. M. Sehgal and S. P. Singh, A theorem on the minimization of a condensing multifunction and fixed points, J. Math. Anal. Appl. 107 (1985), 96-102.

7. _ On random approximations and a random fixed point theorem for set valued mappings, Proc. Amer. Math. Soc. 95 (1985), 91-94.

8. V. M. Sehgal, A simple proof of a theorem of Ky Fan, Proc. Amer. Math. Soc. 63 (1977), 368-369.

9. C. Waters, Ph.D. thesis, University of Wyoming, 1984. 82071

Department of Mathematics, University of Wyoming, LARAmie, Wyoming,

Department of Mathematics, Memorial University of NeWfoundland, St. JOHN'S, NEWFOUNDLAND, CANADA A1C 5S7 